\title{
Theory and simulation of backbombardment in single-cell thermionic-cathode electron guns
}

\author{
J. P. Edelen, ${ }^{*}$ S. G. Biedron, J. R. Harris, and S. V. Milton \\ Colorado State University, Fort Collins, Colorado 80523, USA \\ J. W. Lewellen \\ Los Alamos National Laboratory, Los Alamos, New Mexico 87545, USA
}

(Received 11 February 2015; published 15 April 2015)

\begin{abstract}
This paper presents a comparison between simulation results and a first principles analytical model of electron back-bombardment developed at Colorado State University for single-cell, thermionic-cathode if guns. While most previous work on back-bombardment has been specific to particular accelerator systems, this work is generalized to a wide variety of guns within the applicable parameter space. The merits and limits of the analytic model will be discussed. This paper identifies the three fundamental parameters that drive the back-bombardment process, and demonstrates relative accuracy in calculating the predicted back-bombardment power of a single-cell thermionic gun.
\end{abstract}

DOI: 10.1103/PhysRevSTAB.18.043402

PACS numbers: 79.40.+z, 07.77.Ka, 29.20.Ej

\section{INTRODUCTION}

Thermionic-cathode electron guns have a wide range of uses in particle accelerators and microwave sources. A thermionic cathode's thermal mass prevents rapid changes (relative to microwave frequencies) in temperature, and thus electron emission will occur whenever it is allowed by the surface electric field. Rapid gating is usually accomplished with a grid that can impose a field at the cathode surface and thus control emission [1]. However, a grid also causes effects which impose limitations on machine performance. These include transverse variation in the emitted current, disruption to the beam's distribution in phase space [2], emittance growth [3], and potential beam loss at high current.

These problems can be avoided by operating the cathode in an rf gun without a gating grid, yielding a simple system which is in principle easily scalable to both $\mathrm{cw}$ operation and high currents. However, in these systems electrons emitted late relative to the rf phase do not gain enough energy to exit the gun before the field reverses and will be accelerated back towards the cathode, where they then deposit their kinetic energy in the form of heat. This backbombardment process is undesirable and has adverse effects on machine performance, including cathode damage and a runaway condition in which back-bombardment leads to more cathode heating, leading to more emission, which leads to more back-bombardment [4-7]. While numerous

\footnotetext{
*jedelen@engr.colostate.edu

Published by the American Physical Society under the terms of the Creative Commons Attribution 3.0 License. Further distribution of this work must maintain attribution to the author $(s)$ and the published article's title, journal citation, and DOI.
}

practical techniques exist to mitigate the consequences of back-bombardment [8-13], the physical understanding of how gun design features affect the process is limited to simulation results for specific machines $[5,6]$.

Additionally when designing a thermionic cathode system, estimation of the back-bombardment power is limited to the use of simulation tools such as PARMELA [14], GPT [15], or SPIFFE [16]. Modern computing capabilities have made this process fairly straightforward, however trade-off studies over a wide parameter space using only simulation is cumbersome. Simple analytical tools would allow for an easier evaluation of the back-bombardment power in the initial design process.

Our recent studies of a short-gap gun design operating at many frequencies and beam currents [17] opened the discussion for a more general simulation-based description of the back-bombardment process, but still did not resolve an analytical model that clarifies the underlying physics. To address back-bombardment on a more fundamental level and provide a simple design tool, we developed an analytic theory for back-bombardment in single-cell, thermioniccathode rf guns and conducted a comparative study with simulation.

\section{OVERVIEW OF APPROXIMATIONS}

There are two critical approximations that allow for exact solutions of the back-bombarded particles' energy deposited on the cathode. This is done while maintaining the two fundamental properties of the gun that create backbombardment: (1) the field changing sign and (2) the reversal of the electrons' velocities. These properties are incorporated by using the field profile given by Fig. 1, and the velocity profile given by Eq. (1). While these are not true representations of the fields or the electron velocities 

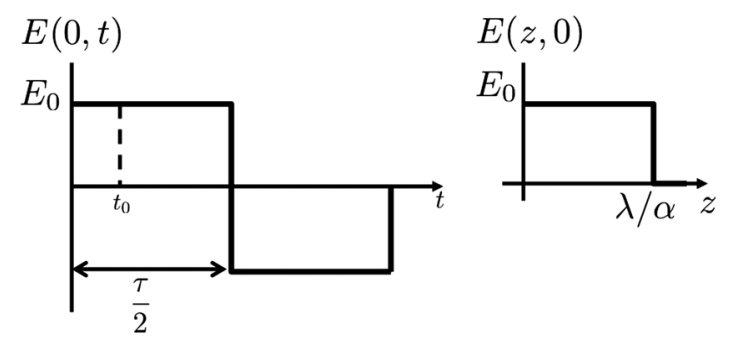

FIG. 1. Temporal (left) and spatial (right) field profiles used for the analytic model. The gap length for the gun is defined in terms of the rf wavelength $\lambda$ as $L_{\text {gap }}=\lambda / \alpha$.

in a rf gun, they serve as a good starting point for the simplified, analytic theory.

Here $t_{0}$ represents the particle emission time, $\tau$ is the $\mathrm{rf}$ period, $E_{0}$ is the peak field, and $1 / \alpha$ is the gap length normalized to the rf wavelength:

$v(z, t)= \begin{cases}v_{\mathrm{eff}} & \text { electrons moving towards exit } \\ 0 & \text { electrons at rest } \\ -v_{\mathrm{eff}} & \text { electrons moving towards cathode }\end{cases}$

Here $v_{\text {eff }}$ is the effective velocity of the electrons, calculated from the average kinetic energy in the gap. It can be shown that the relativistic energy, $\gamma=1 / \sqrt{1-v^{2} / c^{2}}$, is linear with position near the cathode of an $\mathrm{rf}$ gun by $\gamma(z) \approx 1+\frac{E_{0} q}{m_{0} c^{2}} \sin \left(\phi_{0}\right) z$ [18]. The maximum value for $\gamma$ occurs for $\phi_{0}=\pi / 2$; substituting $L_{\text {gap }}$ for $z$ gives $\gamma_{\max }=1+\frac{E_{0} q \lambda}{m_{0} c^{2} \alpha}$. The average value of the relativistic energy, assuming the electrons are initially at rest, is then $\gamma_{\text {ave }}=\left(1+\gamma_{\max }\right) / 2$. The effective velocity for each gun design is then directly calculated from $\gamma_{\text {ave }}$ by $v_{\text {eff }}=c \sqrt{1-1 / \gamma_{\text {ave }}^{2}}$. This is given explicitly in terms of the relevant gun parameters by Eq. (2):

$$
v_{\mathrm{eff}}=c \sqrt{1-\left(1+\frac{q E_{0} \lambda}{2 m_{0} c^{2} \alpha}\right)^{-2}} .
$$

\section{A FIRST PRINCIPLES MODEL OF ELECTRON BACK-BOMBARDMENT}

To study back-bombardment power, we must first determine the boundary between particles that exit the gun and particles that are back-bombarded. This is accomplished by considering a theoretical particle whose emission timing with respect to the rf field is such that it exits the gun with zero energy. A particle that travels at a constant velocity $v_{\text {eff }}$, and is not back-bombarded, has a cavity transit time of $t_{\text {transit }}=\lambda /\left(\alpha v_{\text {eff }}\right)$. A particle that exits with zero energy will see an equal amount of positive and negative field and therefore will have an emission time of $t_{\text {zero }}=\tau / 2-t_{\text {transit }} / 2$. Using the effective velocity computed by Eq. (2), the effective kinetic energy in volts is calculated by solving Eq. (3) for all particles with $t_{0}<\tau / 2-t_{\text {transit }} / 2$ :

$$
K_{\mathrm{eff}}\left(t_{0}\right)=v_{\mathrm{eff}} \int_{t_{0}}^{t_{0}+t_{\text {transit }}} E(t) d t
$$

Note that this is an unphysical model as the velocity of the particle is unchanging with the effective kinetic energy.

For the back-bombardment case, any particle that reaches zero energy before $t_{0}+t_{\text {transit }}$ would then stop, reverse direction, and travel back to the cathode. These particles are emitted when $\tau / 2-t_{\text {transit }} / 2<t_{0}<\tau / 2$ and traverse their path twice. As a result the back-bombarded particles have a different transit time that depends on their emission time, $t_{b b}\left(t_{0}\right)=4\left(\tau / 2-t_{0}\right)$. Substituting $t_{b b}$ for $t_{\text {transit }}$, the effective kinetic energy as a function of emission time for back-bombarded particles can be found using Eq. (3). These results combined give the effective kinetic energy of all particles as a function of emission time (left side of Fig. 2). In order to compute the back-bombardment power, the energy deposited on the cathode as a function of time is required.

The first particle to reach the cathode has zero energy and is emitted last at $t_{0}=\tau / 2$. The last particle to reach the cathode is emitted first, traverses the cavity twice, and arrives at the cathode with an effective kinetic energy of $K_{\text {eff }}^{\max }$, defined by Eq. (5), at time $t=t_{0}+2 t_{\text {transit }}$. This gives a time window for the energy deposited on the cathode of $\tau / 2 \leq t \leq \tau / 2+3 t_{\text {transit }} / 2$, with a range of $0 \leq K_{\text {eff }} \leq K_{\text {eff }}^{\max }$. These points define the linear function that represents the energy deposited on the cathode as a function of time (right side of Fig. 2).

To examine the validity of this model, the relativistic equations of motion are solved numerically for a gun with $\alpha=8$, a peak field of $20 \mathrm{MV} / \mathrm{m}$, and a rf frequency of $1.5 \mathrm{GHz}$. This was used to compute the final kinetic energy

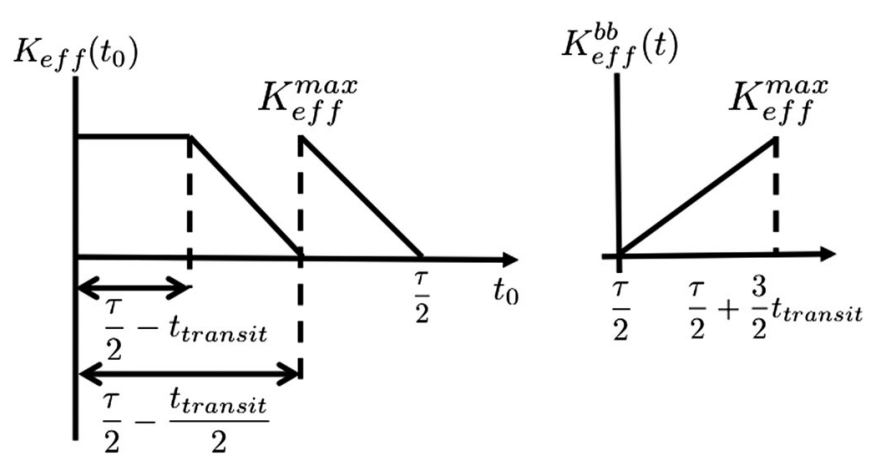

FIG. 2. Left: Final effective kinetic energy as a function of injection time $t_{0}$ for all particles emitted from the cathode. Right: Final effective kinetic energy of the back-bombarded particles arriving at the cathode as a function of time. 


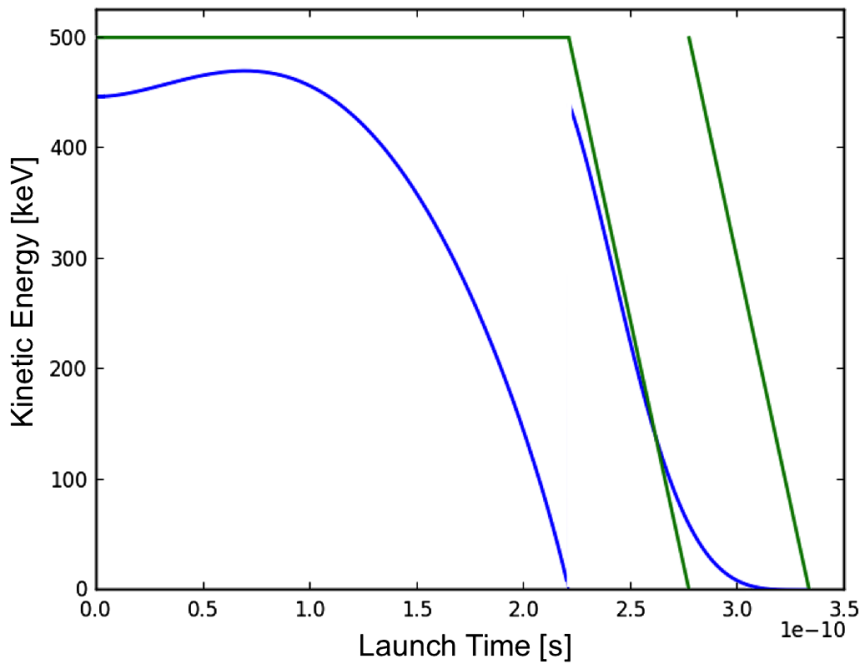

FIG. 3. Blue: Numerical solutions to the relativistic equations of motion for the final energy of a particle emitted at some time $t_{0}$. Green: Final energy as a function in emission time as defined by Fig. 3 .

as a function of emission time and is compared with the model in Fig. 3.

Upon inspection, these two curves appear to be very different. However, when computing back-bombardment power, only the area under the curve to the right of the discontinuity is of importance. Numerically integrating the region to the right of the discontinuity for both curves gives a value of $1.39 \times 10^{-8}$ and $1.41 \times 10^{-8}$ for the blue curve and green curve respectively. These values are very close indicating that the simplified and nonphysical, but computationally tractable, linear model for the energy deposited on the cathode is reasonable for computing the backbombardment power.

Integration of the energy deposited as a function of time (right side of Fig. 2) gives the total effective kinetic energy deposited $K_{\text {eff }}^{\text {dep }}$ on the cathode in units of Volts/Hz as a function of the peak energy:

$$
K_{\mathrm{eff}}^{\mathrm{dep}}=\frac{3 \lambda}{4 \alpha v_{\mathrm{eff}}} K_{\mathrm{eff}}^{\max }
$$

where the peak kinetic energy $\left(K_{\mathrm{eff}}^{\max }\right)$ in volts is defined by Eq. (5),

$$
K_{\text {eff }}^{\max }=\int_{0}^{L_{\text {gap }}} E(z) d z
$$

For a pillbox cavity $K_{\text {eff }}^{\max }=E_{0} \lambda / \alpha$. Because most cavities have more complicated geometries and their axial field maps are computed using numerical codes, analytical solutions to Eq. (5) are not always tractable. The use of a Riemann sum and normalizing to the peak energy gain of a pillbox cavity gives the scaling $K$,

$$
K=\frac{\sum_{i=0}^{N} E(i \Delta z)}{E_{0} N} .
$$

Here $\Delta z=\lambda /(\alpha N)$, where $N$ is the number of data points along the field map (determined by the field map), and $E_{0}$ is the peak value of the axial field. This gives a scaling that takes into account the spatial variation in the field. Multiplying $K_{\text {eff }}^{\text {dep }}$ by the rf frequency and the beam current, substituting $E_{0} \lambda K / \alpha$ for $K_{\text {eff }}^{\max }$, and substituting $c / f$ for $\lambda$, yields the time average back-bombardment power,

$$
P_{\mathrm{ave}}=\frac{3 E_{0} I c^{2}}{4 \alpha^{2} f v_{\mathrm{eff}}} T K
$$

Here $I$ is the average beam current off the cathode, and $T$ is the transit time factor for a pillbox cavity [19]. The transit time factor is a common scaling used in accelerator physics to account for the time variation of the rf field. Because we have chosen a square wave approximation for the time varying component of the field, introduction of the unitless transit time factor helps to adjust for the fact that the simulations are run with a sinusoidal time varying field. For a particle traveling at a constant velocity in a pillbox cavity the transit time factor given by

$$
T=\frac{\sin \left(\pi c / \alpha v_{\text {eff }}\right)}{\pi c /\left(\alpha v_{\text {eff }}\right)}
$$

\section{COMPARISON WITH SIMULATIONS}

Next we compare the results produced by Eq. (7) with simulation data for a representative range of the parameters.

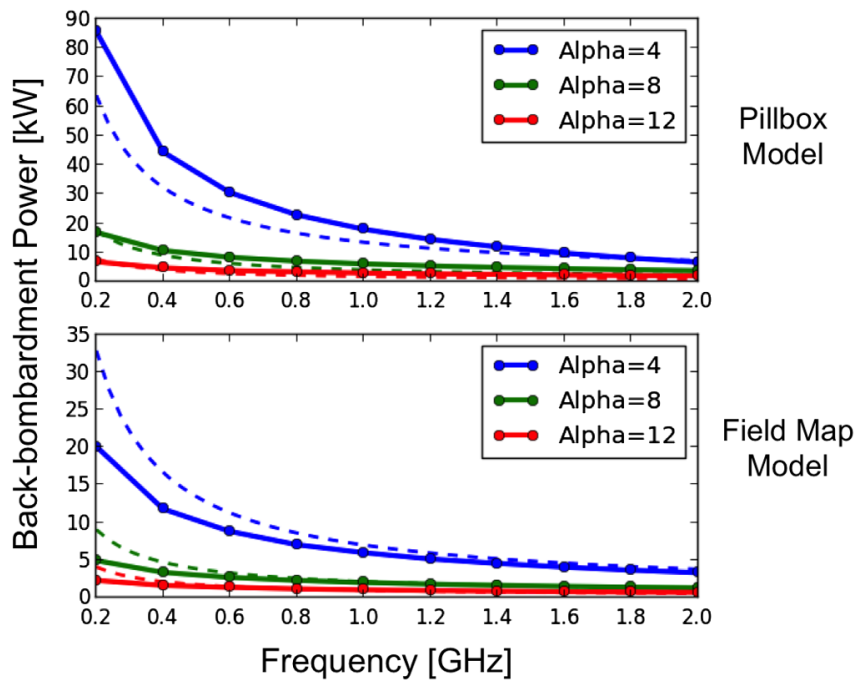

FIG. 4. Comparison of analytic theory (dashed line) with the simulation data (solid line) for the pillbox cavity (top) and singlecell field map (bottom). The peak field for these comparisons is $20 \mathrm{MV} / \mathrm{m}$. 


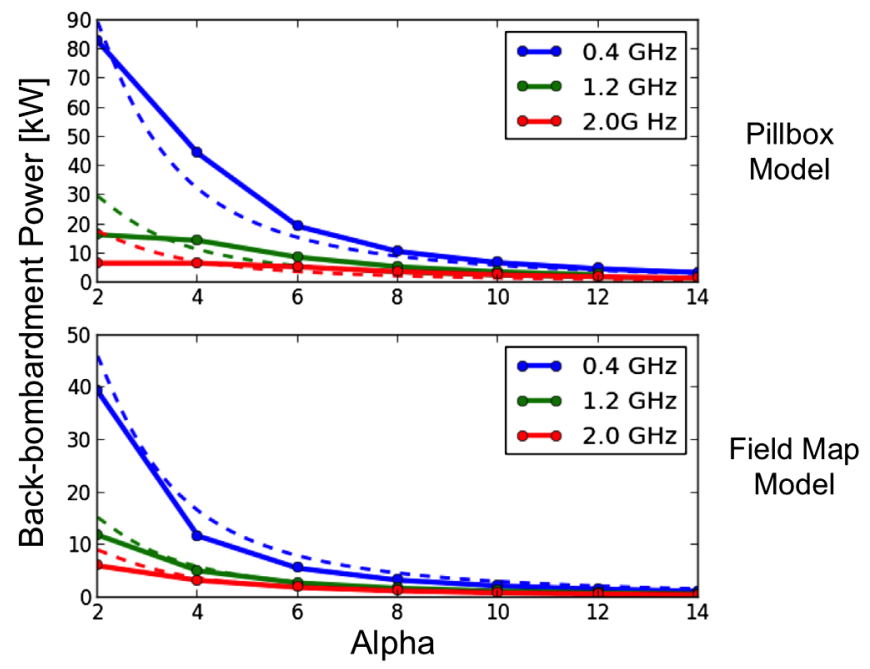

FIG. 5. Comparison of analytic theory (dashed line) with the simulation data (solid line) for the pillbox cavity (top) and singlecell field map (bottom). The peak field for these comparisons was $20 \mathrm{MV} / \mathrm{m}$.

Simulations were performed using a variety of operating frequencies, gap lengths, and peak fields. Two longitudinal field profiles were used: (1) a pillbox $T M_{010}$ mode which is a good approximation for many applications, and (2) a generated longitudinal field map from a representative single cell design [17]. Simulations were performed using SPIFFE [16], a 2.5-D, particle-in-cell, electromagnetic field solver.

For these simulation studies, the peak field was varied from 2 to $30 \mathrm{MV} / \mathrm{m}$, the frequency was varied from $200 \mathrm{MHz}$ to $2 \mathrm{GHz}$, and $\alpha$ was varied from 2 to 14 .

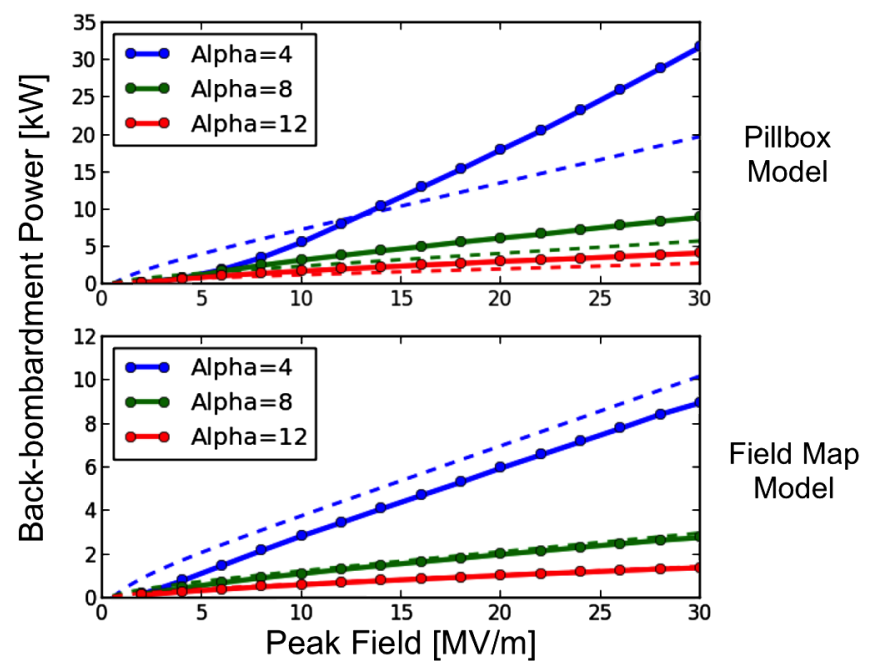

FIG. 6. Comparison of analytic theory (dashed line) with the simulation data (solid line) for the pillbox cavity (top) and single-cell field map (bottom). The rf frequency for these comparisons was $1 \mathrm{GHz}$.
Typically the first cell in a gun has $\alpha=4$ while some configurations call for a longer first cell, $\alpha \approx 3$. We extended our analysis to $\alpha=2$ to include all possible gun configurations. While the simulations were run over a wide range of parameters, only a few representative data sets are presented in Figs. 4-6 to indicate the trends.

The two parameters, frequency and $\alpha$, both have geometrical implications and therefore are addressed first. Figure 4 shows the back-bombardment power as a function of frequency for three values of $\alpha$, and Fig. 5 shows the back-bombardment power as a function of $\alpha$ for three frequencies. It is clear from Figs. 4 and 5 that the analytic model accurately predicts the trends for both the variation with frequency and the variation with $\alpha$.

The third free parameter in the gun design is the peak field. Figure 6 shows the back-bombardment power as a function of peak field for an $\mathrm{rf}$ frequency of $1 \mathrm{GHz}$. Here we see a very good agreement for the field map and a modest agreement for the pillbox. For the pillbox case, the linear trend with field is accurately predicted for higher $\alpha$ and higher peak field, however the slope is not in agreement for low values of $\alpha$. For the field map case, there is good agreement across the board. Figures 4-6 show that frequency, $\alpha$, and peak field all independently affect the backbombardment power. This result is particularly important for initial gun design and optimization as it sheds new light on the fundamental physics of the back-bombardment process.

Using the simulation data and Eq. (7), we computed the magnitude of the difference between simulation and the theory, $D\left(\alpha, f, E_{0}\right)=\left|P_{\text {ave }}\left(\alpha, f, E_{0}\right)-P_{\text {simulation }}\left(\alpha, f, E_{0}\right)\right|$, to quantify how well the model predicts the physics. Table I provides statistics for $D\left(\alpha, f, E_{0}\right)$ for both the pillbox case and the field map case.

This shows that across the entire data set the root mean squared (RMS), and normalized RMS, difference between the theory and the simulation is quite low, indicating that in general the model predicts the physics well. However, large average and peak differences indicate that there are some regions where the model does not agree well with the simulation. Note that the peak difference presented in Table I for the pillbox cavity is larger than the differences shown in Figs. 4-6. This particular case is a gun design with an $\alpha$ of 14, operating at a rf frequency of $2 \mathrm{GHz}$, and a peak field of $30 \mathrm{MV} / \mathrm{m}$. This corresponds to a gap voltage of approximately $320 \mathrm{kV}$. This is a very low voltage

TABLE I. RMS, RMS normalized, average, and peak difference between theory and simulation over the whole range of simulation data.

\begin{tabular}{lcccc}
\hline \hline & $D_{\mathrm{RMS}}[\mathrm{W}]$ & $D_{\mathrm{RMSN}}$ & $D_{\text {ave }}[\mathrm{W}]$ & $D_{\text {peak }}[\mathrm{W}]$ \\
\hline Pillbox case & 270 & 0.0021 & 3700 & 120000 \\
Field map case & 78 & 0.0023 & 1200 & 18000 \\
\hline \hline
\end{tabular}


TABLE II. RMS, RMS normalized, average, and peak difference between theory and simulation where $\psi<200$. This corresponds to $80 \%$ of the data sets.

\begin{tabular}{lcccc}
\hline \hline & $D_{\text {RMS }}[\mathrm{W}]$ & $D_{\text {RMSN }}$ & $D_{\text {ave }}[\mathrm{W}]$ & $D_{\text {peak }}[\mathrm{W}]$ \\
\hline Pillbox case & 100 & 0.0082 & 1700 & 13000 \\
Field map case & 30 & 0.0053 & 420 & 5000 \\
\hline \hline
\end{tabular}

compared to the other configurations and is below the threshold for the model presented in this paper.

Analysis of how the difference function varied with respect to the independent variables showed that no one variable determines whether the error will be low or not. Therefore the parameter, $\psi=\alpha f / E_{0}=c / V_{\text {gap }}$, was introduced. Here $V_{\text {gap }}$ is the gap voltage of the gun defined by $E_{0} L_{\text {gap }}$. Observation of how the difference between simulation and theory varies as a function of $\psi$ showed that $D\left(\alpha, f, E_{0}\right) \propto 1 / \psi=V_{\text {gap }} / c$, with a sharp increase in $D$ for $\psi<200$. This indicated that designs with $\psi<200$ will not agree as well with the theory derived. In other words, the error will increase drastically for designs with a gap voltage less than $1.5 \mathrm{MV}$. This is because for low voltage cases the particle velocities are quite low and varying with time making the constant velocity approximation no longer adequate. Table II shows the same statistics as Table I, excluding designs with $\psi<200$.

Removing cases with $\psi<200$ greatly reduces the RMS, average, and peak difference between simulation and theory. However the normalized RMS has increased slightly. This is likely due to removing cases that have both a large error and a large back-bombardment power and therefore decreasing the normalization value.

\section{CONCLUSIONS}

In conclusion, a first-principles model that predicts backbombardment power in single-cell thermionic-cathode rf guns was derived. This approach was found to accurately predict the trends when compared to simulation results, and furthermore gives order-of-magnitude accuracy for quantitative calculations. Additionally, the $\psi$ parameter was introduced to determine the conditions that cause the theory to break down and helps define where it is useful.

This paper has identified for the first time the three fundamental parameters that drive the back-bombardment process: rf frequency, fractional cavity length, and peak field. In addition, the theory derived here has shown relative accuracy in calculating the predicted back-bombardment power of a single-cell thermionic gun, something that previously required simulations at a minimum.

\section{ACKNOWLEDGMENTS}

We wish to acknowledge Dr. Paul Edelen, Professor of Mathematics at Manchester Community College, for discussions on mathematical modeling.

[1] J. R. Harris and P. G. O'Shea, IEEE Trans. Electron Devices 53, 2824 (2006).

[2] J. R. A. Kighet et al., Nucl. Instrum. Methods Phys. Res., Sect. A 544, 179 (2005).

[3] Y. Zou, H. Li, M. Reiser, and P. G. O'Shea, Nucl. Instrum. Methods Phys. Res., Sect. A 519, 432 (2004).

[4] C. B. McKee and J. M. J. Madey, Nucl. Instrum. Methods Phys. Res., Sect. A 296, 716 (1990).

[5] T. Kii, K. Masuda, S. Amazaki, T. Horii, H. Toku, K. Yoshikawa, H. Ohgaki, and T. Yamazaki, Nucl. Instrum. Methods Phys. Res., Sect. A 483, 310 (2002).

[6] T. Kii, T. Yamaguchi, R. Ikeda, Z.-W. Dong, K. Masuda, H. Toku, K. Yoshikawa, and T. Yamazaki, Nucl. Instrum. Methods Phys. Res., Sect. A 475, 588 (2001).

[7] K. Masuda, T. Horii, S. Amazaki, T. Kii, H. Ohgaki, T. Yamazaki, and K. Yoshikawa, Nucl. Instrum. Methods Phys. Res., Sect. A 483, 315 (2002).

[8] Y. Huang and J. Xie, Measures to alleviate the back bombardment effect of thermionic rf electron gun, http://ieeexplore.ieee.org/xpl/articleDetails.jsp?reload= true\&arnumber; $=164855$.

[9] V. A. Kushnir et al., Simulations of beam performances of the two-cell rf gun, EPAC 1996, http://accelconf.web.cern .ch/accelconf/e96/papers/tupg/tup079g.pdf.

[10] E. Tanabe et al., A novel structure of multi-purpose rf gun, in Proceedings of the 19th International Linear Accelerators Conference, Chicago, IL, 1998 (NTIS, Springfield, VA, 1998).

[11] B. Anderberg et al., A new $3 \mathrm{GHz}$ rf gun structure for MAX-Lab, in Proceedings of the European Particle Accelerator Conference, Vienna, 2000 (EPS, Geneva, 2000).

[12] V.A. Kushnir, High frequency electron guns: Current status, NSC KIPT, Kharkov, Ukraine.

[13] J. M. D. Kowalczyk and J. M. J. Madey, Phys. Rev. ST Accel. Beams 17, 120402 (2014).

[14] L. Young, PARMElA codes, Los Alamos National Laboratory.

[15] S. B. van der Geer and M. J. de Loos, General Particle Tracker code, Pulsar Physics.

[16] M. Borland, SPIFFE (SPace charge and Integration of Forces For Electrons), Advanced Photon Source, 2005.

[17] J. P. Edelen, S. G. Biedron, J. R. Harris, J. W. Lewellen, and S. V. Milton, IEEE Trans. Nucl. Sci. 61, 830 (2014).

[18] K. J. Kim, Nucl. Instrum. Methods Phys. Res., Sect. A 275, 201 (1989).

[19] K. Wille, The Physics of Particle Accelerators an Introduction (Oxford University Press, New York, 2000). 